\title{
Structural and tunneling properties of Si nanowires
}

\author{
E. Montes, ${ }^{1}$ K. Gkionis, ${ }^{1}$ I. Rungger, ${ }^{2}$ S. Sanvito, ${ }^{2}$ and U. Schwingenschlögl ${ }^{1, *}$ \\ ${ }^{1}$ PSE Division, KAUST, Thuwal 23955-6900, Saudi Arabia \\ ${ }^{2}$ School of Physics and CRANN, Trinity College, Dublin 2, Ireland \\ (Received 15 August 2013; published 6 December 2013)
}

\begin{abstract}
We investigate the electronic structure and electron transport properties of Si nanowires attached to $\mathrm{Au}$ electrodes from first principles using density functional theory and the nonequilibrium Green's function method. We systematically study the dependence of the transport properties on the diameter of the nanowires, on the growth direction, and on the length. At the equilibrium Au-nanowire distance we find strong electronic coupling between the electrodes and nanowires, which results in a low contact resistance. With increasing nanowire length we study the transition from metallic to tunneling conductance for small applied bias. For the tunneling regime we investigate the decay of the conductance with the nanowire length and rationalize the results using the complex band structure of the pristine nanowires. The conductance is found to depend strongly on the growth direction, with nanowires grown along the $\langle 110\rangle$ direction showing the smallest decay with length and the largest conductance and current.
\end{abstract}

DOI: 10.1103/PhysRevB.88.235411

PACS number(s): 31.15.A-, 31.15.E-, 62.23.Hj, 61.50.Ah

\section{INTRODUCTION}

One-dimensional medium-sized nanostructured systems such as nanowires (NWs) are believed to be the most important building blocks for the next generation of electronic devices. In particular, Si NWs are attractive candidates due to their compatibility with the existing Si technology. 1,2 Control of the physical properties of NWs can be achieved through changes in the growth conditions and dimensionality., ${ }^{3,4} \mathrm{Si}$ NWs are usually grown by the vapor-liquid-solid technique ${ }^{5,6}$ for varying diameters. Recently the synthesis of diameters below $10 \mathrm{~nm}$ has been achieved. ${ }^{7}$ It has been demonstrated that $\mathrm{Si}$ NWs are rodlike structures with a bulk $\mathrm{Si}$ single crystalline core ${ }^{8}$ and thus grow along well-defined crystalline directions ${ }^{9,10}$ and follow the Si surface reconstruction criteria according to Wulff's minimum energy law, ${ }^{11}$ thereby having a well defined shape in their cross section. ${ }^{12}$ Due to the $s p^{3}$ nature of the Si bonding, the atoms at the surface have dangling bonds, ${ }^{13}$ which need to be passivated in order to ensure chemical stability. To this aim, the synthesized Si NWs are coated with an oxide layer (amorphous oxide formed by exposure to the environment), which is then removed by hydrofluoric acid, resulting in hydrogenated (H-terminated) $\mathrm{Si}$ NWs. ${ }^{14}$

Due to the difficulty of synthesizing Si NWs with various diameters, the optical and electrical properties have been determined experimentally only partially. Theoretical studies can thus fruitfully complement the field. For instance, it has been shown that the electronic structure of Si NWs depends on the growth direction and diameter. ${ }^{15,16}$ The band gap $E_{g}$ is strongly anisotropic and follows the order $E_{g}^{\langle 100\rangle}>E_{g}^{\langle 111\rangle}>$ $E_{g}^{\langle 110\rangle}$ for NWs of comparable diameter ${ }^{17}$ (the superscripts indicate the growth direction). Additionally, the gap decreases exponentially with increasing the diameter from a high value for thin to the bulk Si value for thicker NWs. ${ }^{7,18}$ While bulk Si has an indirect band gap, ${ }^{19} \mathrm{Si}$ NWs usually have a direct band gap, which enables usage as optically active materials for photonics applications. ${ }^{20,21}$

Since the current-voltage $(I-V)$ characteristics are important in circuits design, the transport properties of Si NWs have been widely reported within both theoretical and experimental frameworks. For a system that uses Li electrodes to connect the NW, applying the nonequilibrium Green's function method, the $I-V$ characteristics have been studied as function of the NW geometry ${ }^{17,22}$ and surface modifications created by atomic substitutions and vacancies. ${ }^{7}$ The hole and electron transmission characteristics have been determined for pristine NWs and NWs that contain dopants and vacancies by the Kubo method, recursive Green's function method, and tight binding approaches. ${ }^{23-26} \mathrm{Ge}$ NWs have similar properties to Si NWs and it has been shown that Ge vacancies reduce the thermal conductivity. The transmission coefficients of Si NWs with vacancies have been obtained by the nonequilibrium Green's function method. ${ }^{27}$ The usage of $\mathrm{Au}$ electrodes attached to NWs as well as the surface conduction and current distribution has been investigated by the tight-binding method and Landauer formalism. ${ }^{28}$ Experimentally the $I-V$ characteristics have been determined using two-terminal gate dependent measurements on doped NWs. ${ }^{29}$ It has been shown that it is possible to introduce a high dopant concentration and thereby approach the metallic regime.

In this article we investigate systematically the electron transport properties of $\mathrm{Si}$ NWs attached to semi-infinite $\mathrm{Au}$ electrodes as function of the NW diameter $(D)$, length $(l)$, distance from the electrodes $(d)$, and growth direction. In the first part we study the influence of the Au/NW interface, before addressing the dependence on the diameter. We determine a smooth transition from metallic to semiconducting behavior going from short to large NWs, where at low bias the conductance is via tunneling. For this tunneling transport regime we determine the decay coefficient and compare it to the complex band structure of pristine Si NWs, and analyze the influence of the growth direction and symmetry of the system. Finally we discuss the $I-V$ characteristics for different diameters and growth directions.

\section{METHODS}

We investigate Si NWs with diameters $D=0.6,1.0,1.5$, 2.0 , and $2.5 \mathrm{~nm}$ for the $\langle 100\rangle,\langle 110\rangle$, and $\langle 111\rangle$ principal 


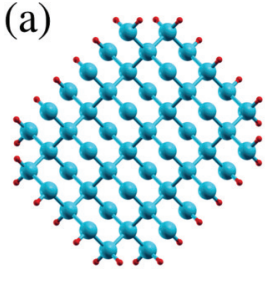

$<100>$

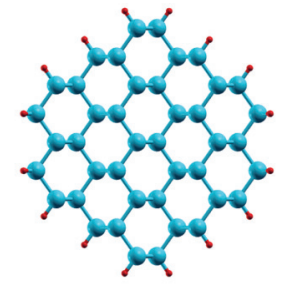

$<110>$

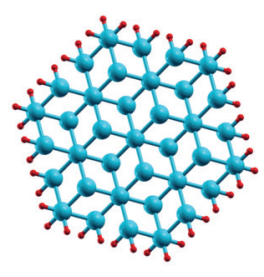

$<111>$

(b)

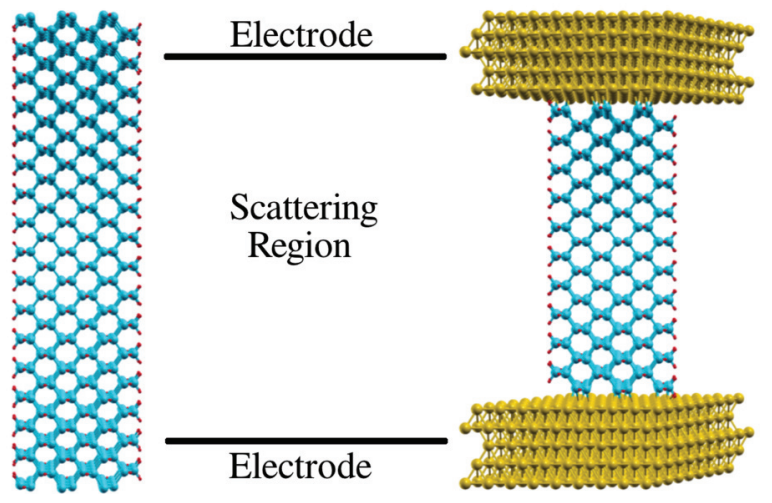

FIG. 1. (Color online) (a) Cross sections of $\langle 100\rangle,\langle 110\rangle$, and $\langle 111\rangle \mathrm{Si}$ NWs. Blue and red spheres represent $\mathrm{Si}$ and $\mathrm{H}$ atoms, respectively. The diameter is $1.5 \mathrm{~nm}$. (b) Configuration of the twoprobe system comprising two electrodes and the central scattering region. For the leads we use both Si NWs (left) and $\mathrm{Au}\langle 111\rangle$ metallic electrodes (right).

growth directions. Figure 1(a) shows the cross section for each growth direction for $D=1.5 \mathrm{~nm}$. We do not consider surface reconstructions and apply the Wulff's law for minimal free energy equilibrium shapes ${ }^{11}$ generalized to NWs, where a cylindrical shape with a core that retains the diamond structure is favored. ${ }^{12}$ Figure $1(\mathrm{~b})$ shows the configurations used in the transport calculations: On the left-hand side a pristine long Si NW is presented, where the outermost units are used as electrodes, while on the right-hand side the $\mathrm{Si} \mathrm{NW}$ is placed between two Au $\langle 111\rangle$ electrodes. All structures are fully relaxed by density functional theory, using the localized orbitals basis set of the SIESTA package, ${ }^{30,31}$ until the forces on all atoms are less than $0.04 \mathrm{eV} / \AA \AA$. A Monkhorst-Pack mesh of $3 \times 3 k$ points in the $x y$ plane (the $z$ axis is the direction of the transport) is used for the calculations with Au electrodes.

In all simulations, double zeta basis sets with polarization functions are employed for all atomic species. The same basis set is used for the transport calculations, with exception of the Au atoms which are described by only one orbital $(6 s)$ in order to reduce the computational cost. The use of an only- $s$ basis for the Au electrodes has been shown to provide reliable results. ${ }^{32,33}$ In all cases the core electrons are described by norm-conserving Troullier-Martins pseudopotentials ${ }^{34}$ and the exchange-correlation potential is calculated within the local density approximation, according to the parametrization proposed by Ceperley and Alder. The real space mesh is set by an equivalent energy cutoff of $150 \mathrm{Ry}$.

Electron transport calculations are performed using the nonequilibrium Green's function approach, as implemented in the SMEAGOL package, ${ }^{35-37}$ which is interfaced with

SIESTA. The system is partitioned in three regions: a left lead $(L)$, a right lead $(R)$, and a central scattering region $(C)$. The leads are modeled as semi-infinite and their influence on the scattering region is taken into account via the left and right self-energies, $\Sigma_{L, R}(E)$. The transmission coefficient through the scattering region is calculated as

$$
T(E)=\operatorname{Tr}\left[G_{C}(E) \Gamma_{L}(E) G_{C}^{\dagger}(E) \Gamma_{R}(E)\right],
$$

where $G_{C}(E)$ is the retarded Green's function of the scattering region and $\Gamma_{L, R}(E)=i\left[\Sigma_{L, R}(E)-\Sigma_{L, R}^{\dagger}(E)\right] .{ }^{27}$ The current through the scattering region is calculated using the Landauer formula

$$
I(V)=\frac{2 e}{h} \int_{-\infty}^{\infty}\left[f\left(E-\mu_{L}\right)-f\left(E-\mu_{R}\right)\right] T(E, V) d E,
$$

where $T(E, V)$ is the transmission coefficient at energy $E$ and bias voltage $V$. Moreover,

$$
f\left(E-\mu_{L, R}\right)=1 /\left\{\exp \left[\left(E-\mu_{L, R}\right) / k_{B} T\right]+1\right\}
$$

is the Fermi-Dirac distribution for the left and right leads with chemical potentials $\mu_{L, R}$.

\section{RESULTS AND DISCUSSION}

We first evaluate the density of states (DOS) and $T(E)$ for a fixed length of Si NWs grown along the $\langle 110\rangle$ direction, as function of the diameter and Au-NW distance. The atomic details of the contact position between $\mathrm{Au}$ and Si NW are relevant to the transmission. ${ }^{38}$ We consider the optimal distance configuration. We investigate the length dependence of the transmission and rationalize the tunneling conductance in terms of the complex band structures of the pristine Si NWs. The results are evaluated for the different growth directions, where the influence of the symmetry of the conducting states is analyzed.

\section{A. Dependence of the DOS and transmission on the Si NW diameter}

We start by presenting the electronic properties of a $\langle 110\rangle$ Si NW with $l=30.72 \AA$ and $D=1.0 \mathrm{~nm}$ attached to $\mathrm{Au}$ electrodes, as a function of the Au-NW distance $d$. The reported $\mathrm{Au}-\mathrm{Si}$ bonding distances for various systems range from 2.30 to $3.50 \AA .{ }^{39,40}$ Our structural relaxations give an optimal geometry with $d=2.30 \AA$, in agreement with previous results. ${ }^{28}$ Using the relaxed geometry as a reference, we construct additional structures by increasing the distance to 3.0, 3.5, and 4.0 A. Such variations might appear for electrodes with rough surfaces or for $\mathrm{Si}$ NWs with different terminations. To observe the effect of changes in $d$ on the electronic structure we calculate the projected DOS for a Si atom that belongs to the $\mathrm{Si} \mathrm{NW}$ plane in contact with the $\mathrm{Au}\left(\mathrm{Si}_{1}\right)$ and for a $\mathrm{Si}$ atom in the middle of the NW $\left(\mathrm{Si}_{2}\right)$. The results are shown in Fig. 2. While the $\mathrm{Si}_{2}$ atom shows a gap around the Fermi energy $\left(E_{F}\right)$, the $\mathrm{Si}_{1}$ atom has a rather high and smooth DOS for all energies. This indicates metallicity of the $\mathrm{Si}$ interface layers and therefore a good contact between $\mathrm{Au}$ and the $\mathrm{Si}$ NW. As $d$ progressively increases the electronic coupling to the Si NW decreases and, as a result, the peaks in the $\mathrm{Si}_{1}$ DOS 

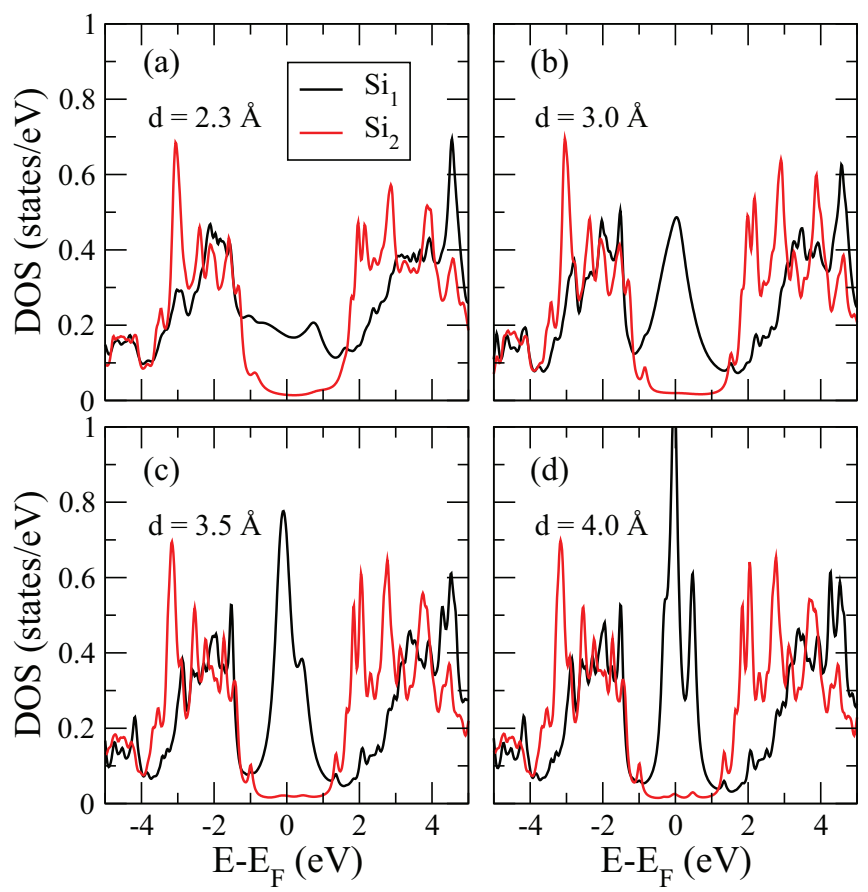

FIG. 2. (Color online) Projected DOS for the Au-NW-Au system ( $D=1.0 \mathrm{~nm}, l=30.72 \AA$ ) for different Au-NW distances $d$. The $\mathrm{Si}_{1}$ atom belongs to the plane in contact with $\mathrm{Au}$, while $\mathrm{Si}_{2}$ is located approximately in the middle of the $\mathrm{Si} \mathrm{NW}$ between the Au planes.

become sharper. This is especially notable for the peak near $E_{F}$, which stems from the fact that pulling apart the NW from the Au leaves the $s p^{3}$ surface $\mathrm{Si}$ atoms unsaturated. The DOS of $\mathrm{Si}_{2}$ is also smoother for smaller $d$ but overall preserves its shape for increasing $d$. We note that $E_{F}$ for all values of $d$ is approximately at midgap of the $\mathrm{Si}_{2}$ DOS, so that at low bias the short Si NWs are expected to conduct in a tunneling regime, where the conductance is expected to decay exponentially with increasing length.

Next we investigate the dependence of the transport properties on the diameter $D$. To this aim we calculate $T(E)$ for a range of diameters of Si NWs grown along the $\langle 110\rangle$ direction, at the optimal $d$ and for $l=30.72 \AA$. To disentangle interface effects from inherent changes in the transmission, we compare $T(E)$ for a setup where Si NWs are used as semiinfinite leads (NW-NW) to the Au electrode case (Au-NW-Au), see Fig. 1(b). $T(E)$ for the NW-NW system corresponds to the number of open channels (the number of right-propagating states at a given energy) in the infinite Si NWs and therefore represents an upper limit for a finite Si NW. In Fig. 3 we present $T(E)$ for the two systems. The transmission is higher for the NW-NW system, since it is homogeneous and thus lacks scattering at the interface, in contrast to the Au-NW-Au system. There is, however, a good correspondence between $T(E)$ for the two systems, also with respect to the magnitude and position of the transmission gap. In the high conductance regions away from the energy gap $T(E)$ for the Au-NW-Au system is reduced by a factor of up to 2 with respect to the conductance of the NW-NW system. The fact that the transmission reduction across the two Au-NW interfaces is only of about 2 indicates that overall the contact resistance is

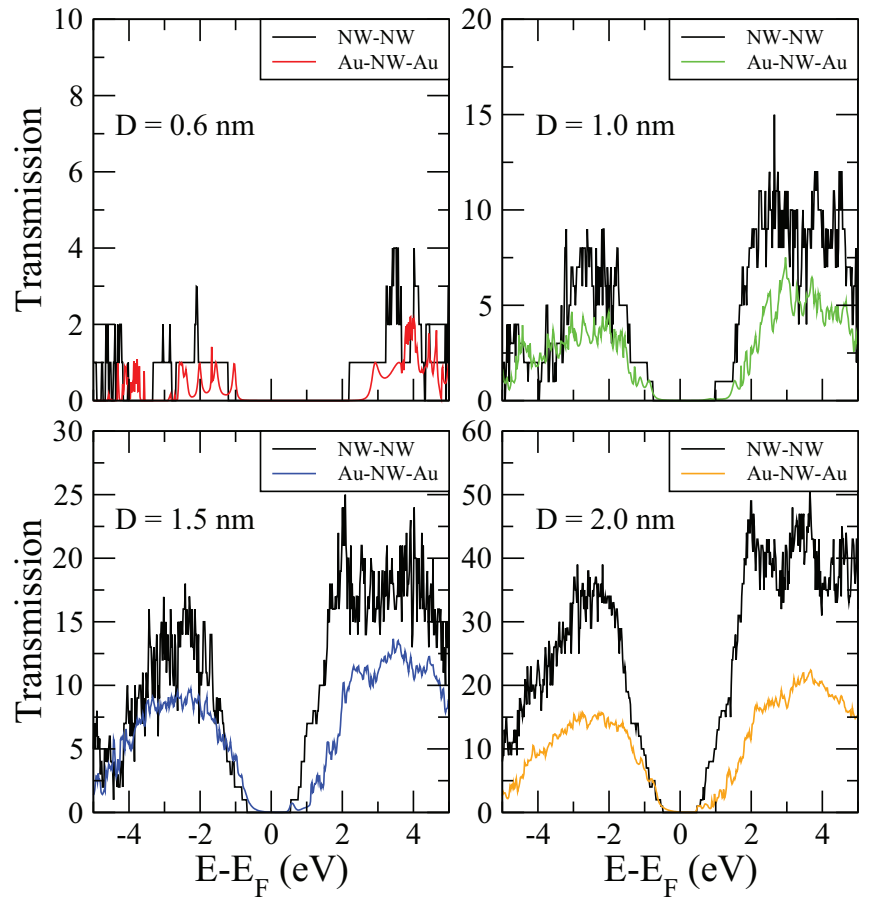

FIG. 3. (Color online) Transmission coefficient for different diameters of the Si NW: $0.6,1.0,1.5$, and $2.0 \mathrm{~nm}(l=30.72 \AA$ and $d=2.3 \AA$ ). The growth direction is $\langle 110\rangle$.

rather small. This is consistent with the metallic DOS of the interface $\mathrm{Si}$ atoms, see Fig. 2(a), which originates from the good electronic coupling to the Au electrodes. For the thinnest Si NW the transmission of the Au-NW-Au system shows a peaked structure, indicating that it behaves like a molecule due to its finite length. For the thicker Si NWs the transmission is a rather smooth function. This is also reflected by the larger number of open channels in the valence and conduction bands of the infinite Si NWs, which increases from well below 10 for $D=0.6 \mathrm{~nm}$ to about 40 for $D=2.0 \mathrm{~nm}$. Since the electronic states are mainly bulklike, in a first approximation the number of open channels is proportional to $D^{2}$. In Fig. 4 the increase of $T(E)$ for larger diameters in the Au-NW-Au system is clearly visible. The transport gap around $E_{F}$ is shown in Fig. 4 as a function of $D$. It can be seen that it is reduced exponentially for increasing $D$ towards the bulk $\mathrm{Si}$ value, in agreement with the literature. ${ }^{18}$

\section{B. Length dependence of the transmission}

We move on to the investigation of the dependence of the transmission on the length of the NW. In Fig. 5 the transmission is shown for different lengths of the $\langle 110\rangle \mathrm{Si} \mathrm{NW}$ with $D=$ $1.0 \mathrm{~nm}$ and $d=2.3 \AA$. For short NWs there is a metallic state induced by the contact with $\mathrm{Au}$, which also has been found for Li electrodes. ${ }^{22}$ For longer NWs a semiconducting state is found, allowing usage of the system in electronic devices. Within the gap the transmission occurs via tunneling. In order to determine the behavior of $T(E)$ in this regime we calculate the complex band structure, ${ }^{41,42}$ which allows us to find the dominant contributions to $T(E)$. For tunneling transport we 


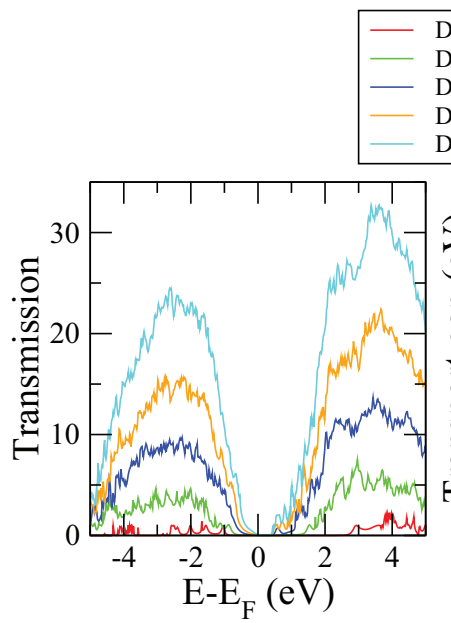

$$
\begin{aligned}
& \mathrm{D}=0.6 \mathrm{~nm} \\
& \mathrm{D}=1.0 \mathrm{~nm} \\
& \mathrm{D}=1.5 \mathrm{~nm} \\
& \mathrm{D}=2.0 \mathrm{~nm} \\
& \mathrm{D}=2.5 \mathrm{~nm}
\end{aligned}
$$

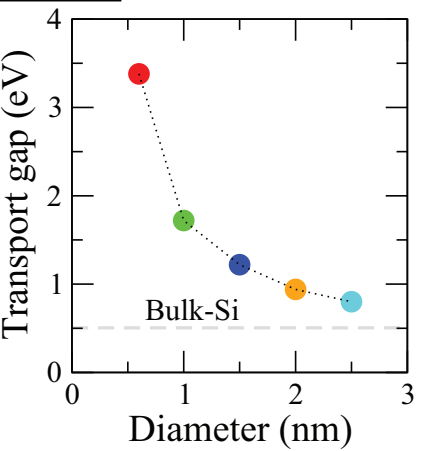

FIG. 4. (Color online) Transmission coefficient for the Au-NWAu system $(l=30.72 \AA$ and $d=2.3 \AA)$ for different diameters. Transport gap around $E_{F}$ as a function of the diameter: The dashed line is the value for bulk $\mathrm{Si}$.

have

$$
T(E)=T_{0}(E) e^{-2 \kappa(E) l},
$$

where $T_{0}$ is a constant and corresponds to the extrapolated transmission for $l=0$ and $\kappa$ is the decay or damping coefficient. By calculating the transmission for two lengths $l_{1}$ and $l_{2}$ the damping coefficient can be obtained as

$$
\kappa(E)=\frac{1}{2\left(l_{2}-l_{1}\right)} \ln \frac{T_{1}(E)}{T_{2}(E)} .
$$
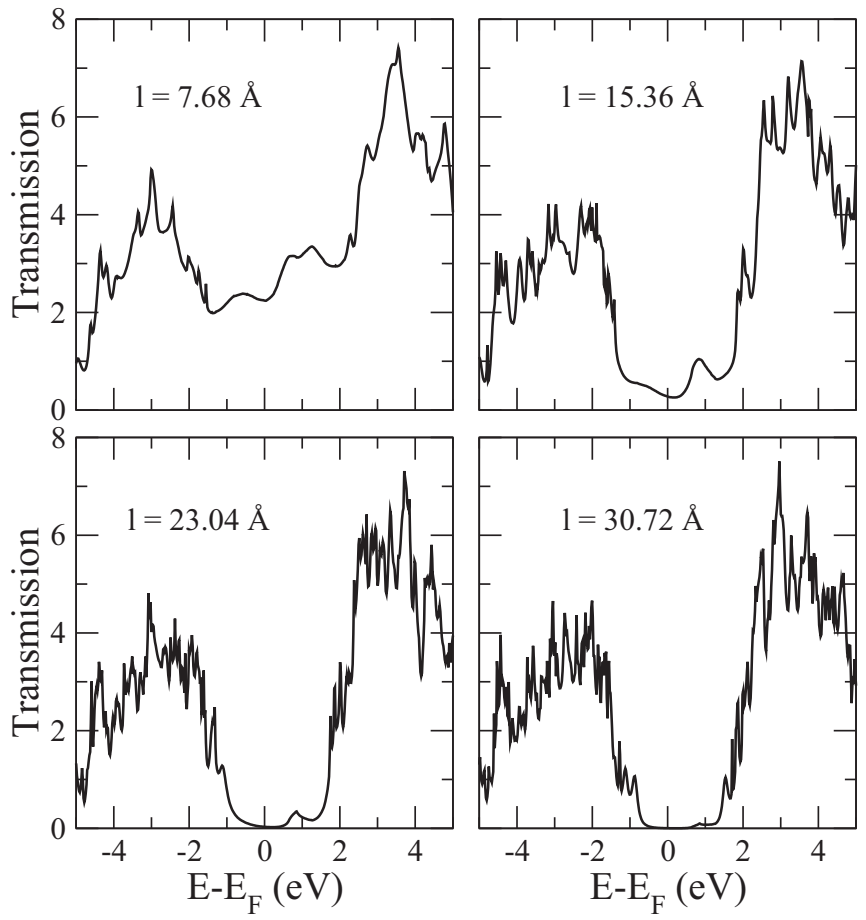

FIG. 5. Transmission coefficient for the Au-NW-Au system for lengths $7.68,15.36,23.04$, and $30.72 \AA(D=1.0 \mathrm{~nm}$ and $d=2.3 \AA)$ in the direction $\langle 110\rangle$. There is a smooth transition from metallic to semiconducting behavior as the NW length increases.

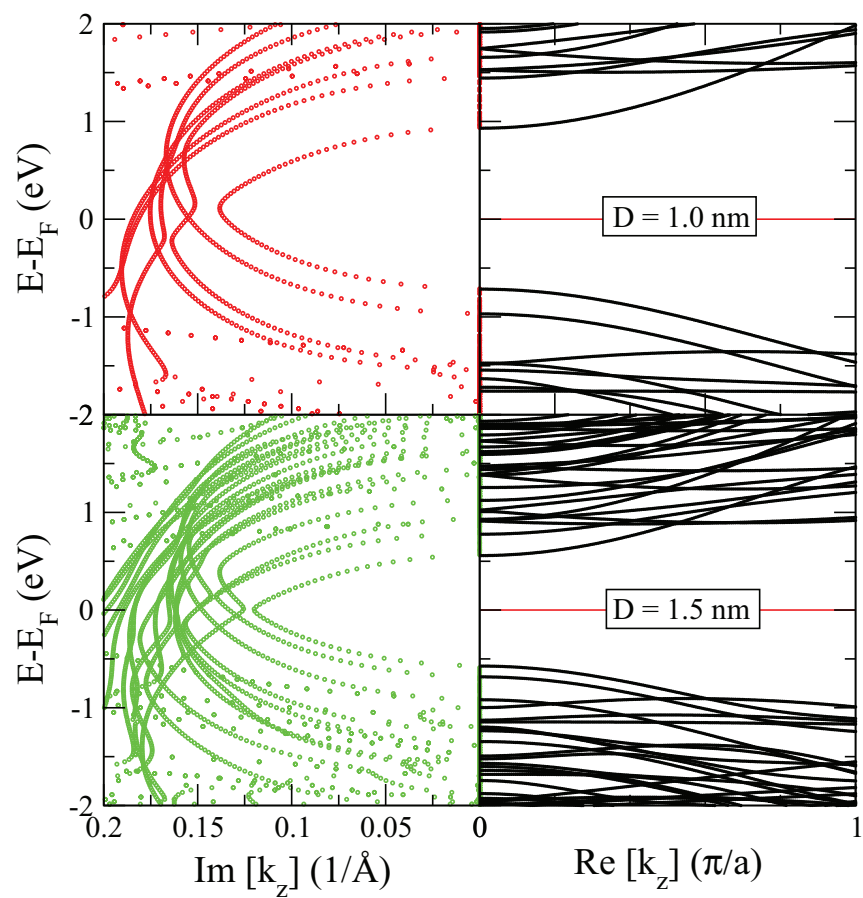

FIG. 6. (Color online) Complex (left) and real (right) band structure for a Si NW with $\langle 110\rangle$ growth direction $(D=1.0$ and $1.5 \mathrm{~nm}$ ).

It can also be approximated by application of the Bloch theorem for infinite periodic NWs by the inverse dispersion relation, where for a given energy one computes all possible $k_{z}$ values that correspond to eigenstates of the system. If $k_{z}(E)$ is real then the state is propagating along the $z$ direction, whereas if $\operatorname{Im}\left[k_{z}(E)\right]>0\left\{\operatorname{Im}\left[k_{z}(E)\right]<0\right\}$ then it is an exponentially decaying (increasing) state. For infinitely extended systems the imaginary solutions of the band structure represent an unreasonable physical behavior, since they blow up exponentially. For systems of finite size the complex wave vectors have a relevant physical meaning, since they determine how an electron with a given energy tunnels trough a finite length of the material. Within the gap region there are no propagating states and $T(E)$ thus is dominated by the state with the slowest decay, which corresponds to the smallest $\operatorname{Im}\left[k_{z}(E)\right]$ at that energy, $\operatorname{Im}\left[k_{z}(E)\right]_{\min }$. We therefore have $\kappa(E) \approx \operatorname{Im}\left[k_{z}(E)\right] .{ }^{42} \mathrm{~A}$ detailed discussion of the complex band analysis for tunneling systems is given in Ref. 43 .

In Fig. 6 we present the complex and real solutions for $\langle 110\rangle$ Si NWs with different diameters. The real band structure shows the expected direct band gap. Within the gap region between the valence states (the highest occupied molecular orbital, HOMO) and conduction states (the lowest unoccupied molecular orbital, LUMO) there is no real band ( $k_{z}$ is purely imaginary). Note that at each energy there are many complex decay vectors. However, for the transmission only those with the smallest values are relevant, since the others have negligible tunneling probability. In the fundamental gap region the smallest values of the complex band structure are given by a semielliptical curve, see Fig. 6(a).

In Fig. 7 we present $T(E)$ for the $\langle 110\rangle$ Au-NW-Au system for various lengths on a logarithmic scale to emphasize the 


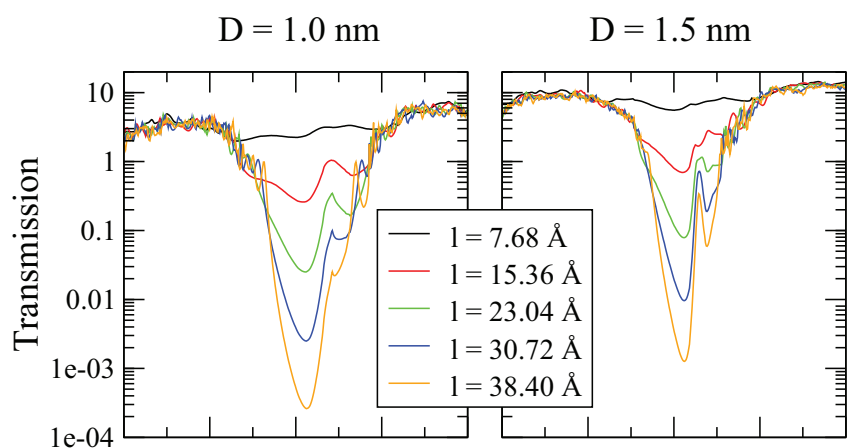

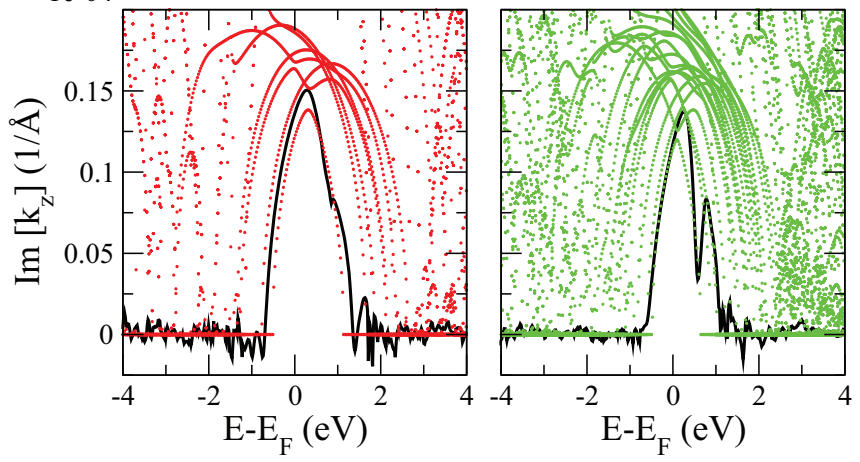

FIG. 7. (Color online) Transmission coefficient (top), complex band structure (bottom, color), and damping coefficient (bottom, black) for NWs with diameters $D=1.0 \mathrm{~nm}$ (left) and $D=1.5 \mathrm{~nm}$ (right), and $\langle 110\rangle$ growth direction.

values in the tunneling regime around $E_{F}$. We calculate $\kappa(E)$ using an exponential fit of the transmission versus length to Eq. (5). The standard error of the fit at the Fermi energy is always below $0.005 / \AA$. The resulting decay coefficient is shown as black line in the bottom part of Fig. 7 for $D=1.0$ and $D=1.5 \mathrm{~nm}$. The colored dots correspond to the complex band structure $\operatorname{Im}\left[k_{z}(E)\right]$ from Fig. 6 . It can be seen that we have in good approximation $\kappa(E) \approx \operatorname{Im}\left[k_{z}(E)\right]_{\min }$. This demonstrates that the transmission is indeed in the tunneling regime for energies within the gap and follows the model.

In order to analyze the symmetry of the HOMO and LUMO states we plot the isosurface electronic wave functions of the $\langle 110\rangle$ Si NWs with $D=1.0 \mathrm{~nm}$ in the top part of Fig. 8 . Positive lobes are indicated in blue and negative lobes in red. The HOMO mainly consist of Si $3 p_{z}$ states with an evenly distributed weight across the area. The LUMO is more located on the surface, where the main contribution also comes from the $\mathrm{Si} 3 p_{z}$ states. The equal symmetry of the HOMO and LUMO is the origin of the anticrossing semielliptical behavior indicate by arrows in Fig. 6 . For the $D=1.5 \mathrm{~nm} \mathrm{NW}$ the symmetry of the HOMO and LUMO is analogous.

When connected to Au electrodes we expect the dominant contributions to $T(E)$ to come from the HOMO and LUMO states for energies around $E_{F}$. To verify this expectation we address the transmission channel with the largest eigenvalue. The transmission eigenchannels are obtained by diagonalizing the transmission matrix, where the state contributing most to the total transmission has the largest eigenvalue. For each eigenvalue there is a corresponding wave function, originating either from the left or the right electrode..$^{44}$ For states from the left electrode we plot the transmission eigenchannel wave
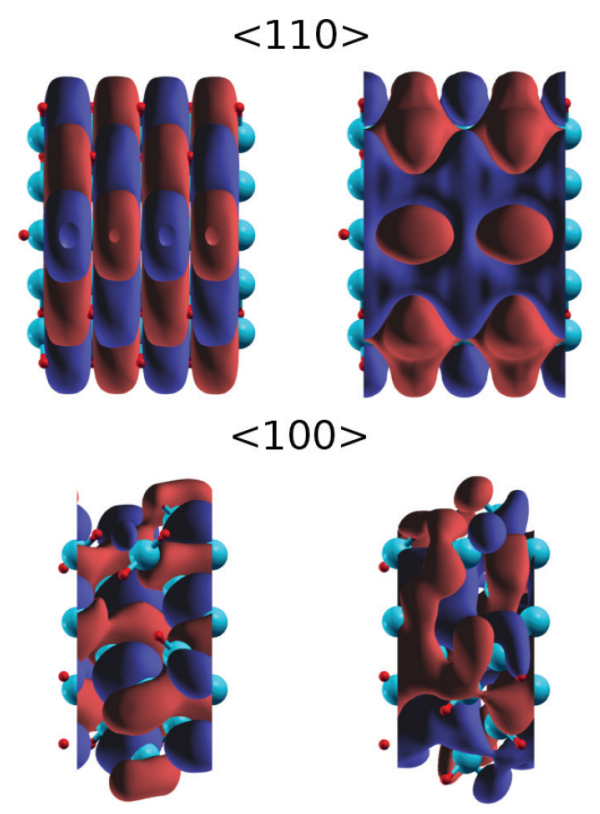

$<111>$
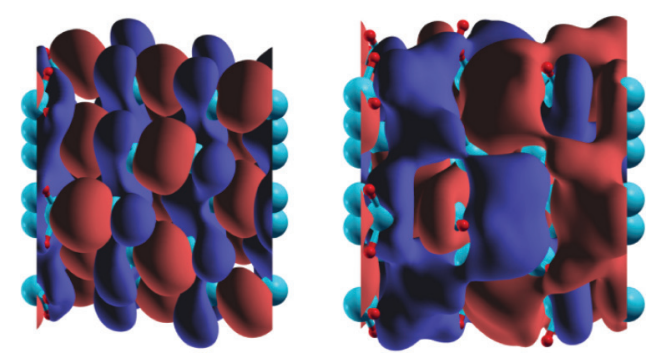

FIG. 8. (Color online) Isosurface wave functions for the (left) HOMO and (right) LUMO. The NW growth direction is (top) $\langle 110\rangle$, (middle) $\langle 100\rangle$, and (bottom) $\langle 111\rangle$, and the diameter is $D=1.0 \mathrm{~nm}$.

functions in Fig. 9 for three energies: $-0.87 \mathrm{eV}$ [Fig. 9(a)], $0.16 \mathrm{eV}$ [Fig. 9(b)], and $1.53 \mathrm{eV}$ [Fig. 9(c)]. In the central region of the Si NW the transmission wave functions have the character of the HOMO and LUMO for -0.87 and $1.53 \mathrm{eV}$, respectively, see Figs. 8(a) and 8(b). At these energies the system exhibits metallic conductance and, in fact, the transmission wave functions extend over the whole system. In the region close to the Au electrodes the transmission wave functions have different character due to strong hybridization of the Au and Si states. The Au induced states extend only over a part of the Si NW. For an energy in the gap [Fig. 9(b)] the transmission wave function decays exponentially in the NW from left to right, where for the chosen energy the character of the decaying state is HOMO-like. We note that the amplitude at the left boundary of the Si NW is rather large due to the good contact to the Au electrode, reflecting the metallic DOS in this region as discussed in the previous sections.

\section{Different growth directions}

We now generalize the presented results to the $\langle 100\rangle$ and $\langle 111\rangle$ growth directions. We find that the optimum distance between $\mathrm{Au}$ and the $\mathrm{Si} \mathrm{NW}$ is equal for all cases. The reduction of the transmission and electronic gaps with the diameter $D$ is also analogous for all directions, as is the critical 


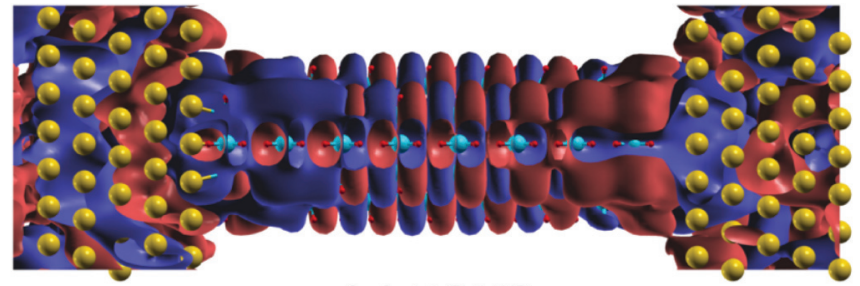

(a) HOMO

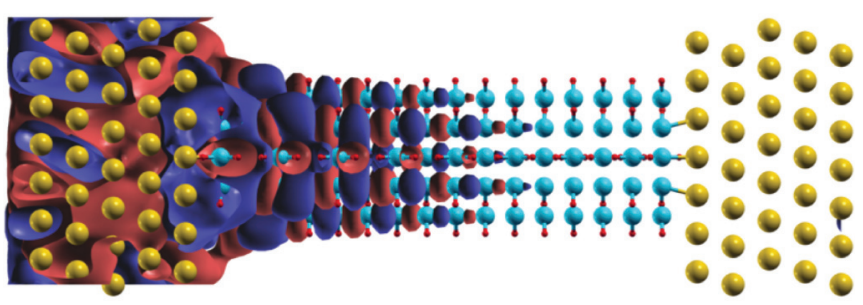

(b) Gap region

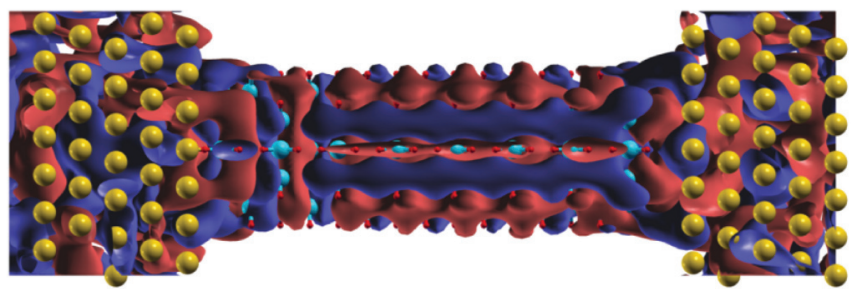

(c) LUMO

FIG. 9. (Color online) Isosurface transmission wave functions for the (a) HOMO, (b) gap region, and (c) LUMO, for the Au-Si NW-Au system with Si NW growth direction $\langle 110\rangle$.

length $l$ above which the system with Au electrodes becomes semiconducting.

We plot the real and complex solutions for the $\langle 100\rangle$ and 〈111〉 Si NWs with diameter $D=1.0 \mathrm{~nm}$ in Fig. 10. The real band structures have the expected band gap. The transmission and damping coefficients are addressed in Fig. 11. For the $\langle 100\rangle$ growth direction the band gap is direct. However, the states with the lowest $\operatorname{Im}\left[k_{z}(E)\right]$ in the midgap region are not the HOMO and LUMO states but rather HOMO-1 and $\mathrm{LUMO}+1$. The tunneling therefore is expected to have contributions from HOMO-1, HOMO, LUMO, and $\mathrm{LUMO}+1$, depending on the energy. The damping coefficient is again in good agreement with $\operatorname{Im}\left[k_{z}(E)\right]_{\min }$. The HOMO and LUMO wave functions for the infinite NW are presented in middle part of Fig. 8. The HOMO is mainly characterized by $\mathrm{Si} 3 p_{x}$ and $\mathrm{Si} 3 p_{y}$ states with the weight distributed evenly across the NW. The LUMO, on the other hand, has a different spatial distribution with a high level of delocalization and contributions of states along all principal directions, namely $\mathrm{Si} 3 p_{x}, \mathrm{Si} 3 p_{y}$, and $\mathrm{Si} 3 p_{z}$. Due to the different symmetries, the HOMO and LUMO complex band structures do not anticross, whereas HOMO-1 and LUMO +1 have the same symmetry, resulting in an anticrossing. The overall transmission is reduced with respect to the $\langle 110\rangle$ orientation.

For the $\langle 111\rangle$ growth direction the main difference is that $\operatorname{Im}\left[k_{z}(E)\right]_{\min }$ does not follow a semielliptical shape and that there is a crossing between the complex band originating from the HOMO and the one originating from the LUMO. This
TABLE I. Effective mass and band gap for different NW orientations with diameter $D=1.0 \mathrm{~nm}$ ( $m_{e}$ is the electron mass).

\begin{tabular}{lccc}
\hline \hline & $\langle 100\rangle$ & $\langle 111\rangle$ & $\langle 110\rangle$ \\
\hline Effective mass & $0.4292 m_{e}$ & $0.1688 m_{e}$ & $0.1202 m_{e}$ \\
Band gap & $2.6856 \mathrm{eV}$ & $2.2351 \mathrm{eV}$ & $1.7216 \mathrm{eV}$ \\
\hline \hline
\end{tabular}

indicates that the HOMO and LUMO have different symmetry. Wave function plots for the infinite Si NW are presented in the bottom part of Fig. 8. Both the HOMO and LUMO are characterized by a combination of $\mathrm{Si} 3 p_{x}, \mathrm{Si} 3 p_{y}$, and $\mathrm{Si} 3 p_{z}$ states.

Comparing the decay coefficients of the different orientations, we find for similar diameters for the $\langle 110\rangle \mathrm{Si} \mathrm{NW}$ a value of $0.15 / \AA$ at midgap, whereas for the other orientations the value is larger by about a factor 2 . This implies that the tunneling conductance for the $\langle 110\rangle$ growth direction is significantly larger than for the other orientations. The origin of the different decay coefficients is the different effective mass of the conduction and valence electrons for the different growth directions, where the effective mass of the $\langle 110\rangle$ direction is the smallest, corresponding also to the smallest gap, see Table I. The smaller effective mass of the $\langle 110\rangle$ orientation also implies that the mobility can be expected to be the largest.

\section{I-V characteristics}

Finally, we present the $I-V$ characteristics of the Au-NWAu system for NWs of diameters $D=1.0$ and $1.5 \mathrm{~nm}$, grown in the $\langle 110\rangle$ direction, see Fig. 12(a). As expected, the NW of larger diameter has a higher current for any given voltage. This is in agreement with the observed transmission dependence on

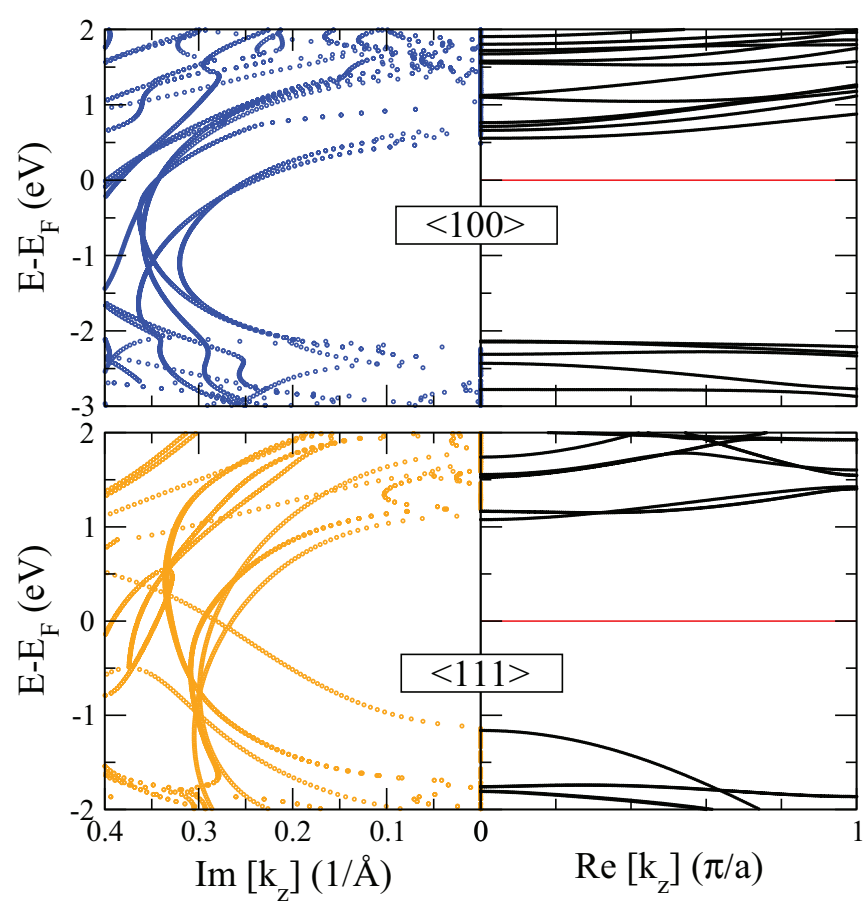

FIG. 10. (Color online) Complex and real band structures for Si NWs with $D=1.0 \mathrm{~nm}$ and growth directions (top) $\langle 100\rangle$ and (bottom) $\langle 111\rangle$. 

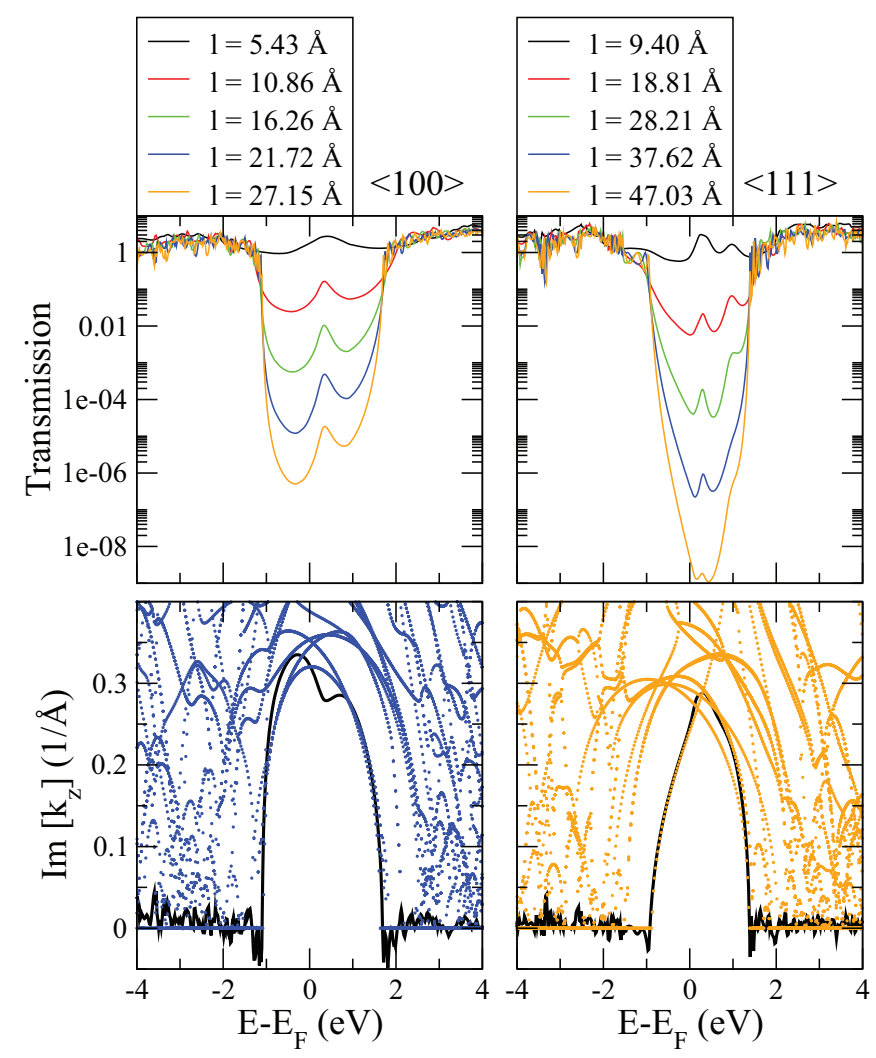

FIG. 11. (Color online) Transmission coefficient (top), complex band structure (bottom, color), and damping coefficient (bottom, black) for NWs with growth directions $\langle 100\rangle$ (left) and $\langle 111\rangle$ (right), and diameter $D=1.0 \mathrm{~nm}$.

the diameter, see above, since the current is the integral of the transmission over the bias window (energy region that contributes to the current integral). A similar trend is also observed when comparing the $I-V$ characteristics for NWs of different growth directions at a constant diameter $D=1.0 \mathrm{~nm}$, see Fig. 12(b): For a given bias voltage the current follows the order $I^{\langle 110\rangle}>I^{\langle 111\rangle}>I^{\langle 100\rangle}$, which confirms the trend reported in the literature. ${ }^{17}$ The gap size order is inverted $E_{g}^{\langle 100\rangle}>E_{g}^{\langle 111\rangle}>E_{g}^{\langle 110\rangle}$.

\section{CONCLUSIONS}

We have systematically studied the dependence of the electron transport properties of Si NWs attached to $\mathrm{Au}\langle 111\rangle$

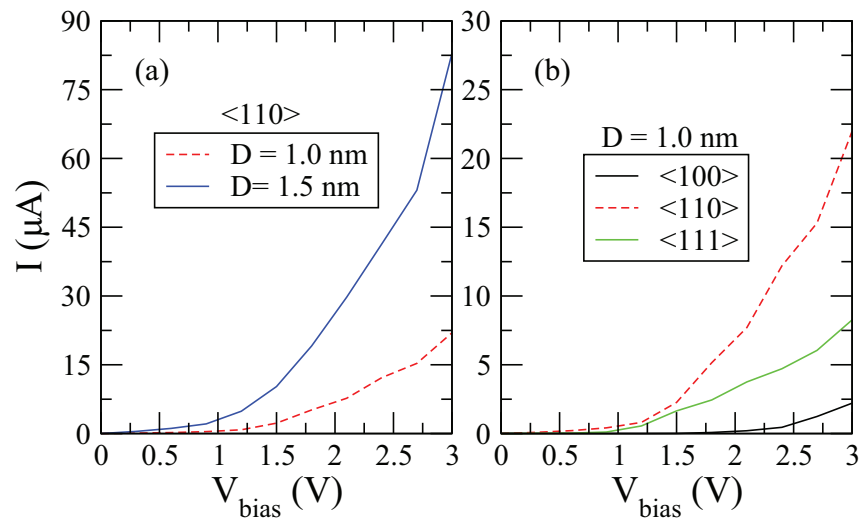

FIG. 12. (Color online) $I-V$ characteristics for Au-NW-Au systems: (a) $\langle 110\rangle \mathrm{Si}$ NWs with diameters $D=1.0$ and $1.5 \mathrm{~nm}$. (b) $\mathrm{Si}$ NWs with $D=1.0 \mathrm{~nm}$, grown in the three principal directions.

electrodes on their diameter, length, and growth direction. Such knowledge is essential for the design of NW-based electrical devices such as nanotransistors. The band gaps of the modeled Si NWs depend both on the growth direction and the diameter. An increase of the latter results in larger cross sections, which in turn induces an enhanced transmission. The relaxed distance of $2.3 \AA$ between the Si NW and Au electrodes is in agreement with previously reported results. At this distance we find a low contact resistance to Au, indicating good electronic coupling. An increase of the distance, which might appear for uneven $\mathrm{Au}$ surfaces, leads to a weakening of the Au-NW coupling and results in more states around $E_{F}$ as a consequence of the formation of dangling bonds on the $\mathrm{Si}$ atoms in the vicinity of the Au. For NWs longer than four unit cells the transmission around $E_{F}$ is found to be of tunneling type, with $E_{F}$ approximately in the middle of the NW electronic gap. For the tunneling regime of the electron transport we find that $\langle 110\rangle$ Si NWs have the largest conductance and smallest decay with the length for fixed diameter. This is due to the smaller effective mass of both the conduction and valence electrons as well as the smaller band gap. Finally, the transmission and band gap dependencies on the NW diameter and growth direction are reflected in the $I-V$ characteristics of the modeled devices. We find a current order of $I^{\langle 110\rangle}>I^{\langle 111\rangle}>I^{\langle 100\rangle}$ for the different growth directions. *udo.schwingenschlog1@kaust.edu.sa

${ }^{1}$ P. Peercy, Nature (London) 406, 1023 (2000).

${ }^{2}$ Y. Cui and C. M. Lieber, Science 291, 851 (2001).

${ }^{3}$ C. M. Lieber, Solid State Commun. 107, 607 (1998).

${ }^{4}$ T. Palacios, Nature (London) 481, 152 (2012).

${ }^{5}$ R. S. Wagner and W. C. Ellis, Appl. Phys. Lett. 4, 89 (1964).

${ }^{6}$ J. D. Weswater, D. P. Gosain, S. Tomiya, S. Usui, and H. Ruda, J. Vac. Sci. Technol. B 15, 554 (1997).

${ }^{7}$ D. D. D. Ma, C. S. Lee, F. C. K. Au, S. Y. Tong, and S. T. Lee, Science 299, 1874 (2003).
${ }^{8}$ Y. F. Zhang, L. S. Liao, W. H. Chan, S. T. Lee, R. Sammynaiken, and T. K. Sham, Phys. Rev. B 61, 8298 (2000).

${ }^{9}$ Y. Wu, Y. Cui, L. Huynh, C. Barrelet, D. Bell, and C. Lieber, Nano Lett. 4, 433 (2004).

${ }^{10}$ A. Lugstein, M. Steinmair, Y. J. Hyun, G. Hauer, P. Pongratz, and E. Bertagnolli, Nano Lett. 8, 2310 (2008).

${ }^{11}$ L. D. Marks, Rep. Prog. Phys. 57, 603 (1994).

${ }^{12}$ Y. Zhao and B. I. Yakobson, Phys. Rev. Lett. 91, 035501 (2003).

${ }^{13}$ R. Rurali, Rev. Mod. Phys. 82, 427 (2010). 
${ }^{14}$ F. M. Ross, J. Tersoff, and M. C. Reuter, Phys. Rev. Lett. 95, 146104 (2005)

${ }^{15}$ X. Zhao, C. M. Wei, L. Yang, and M. Y. Chou, Phys. Rev. Lett. 92, 236805 (2004).

${ }^{16}$ P. W. Leu, B. Shan, and K. Cho, Phys. Rev. B 73, 195320 (2006).

${ }^{17}$ M. F. Ng, L. Zhou, S. W. Yang, L. Y. Sim, V. B. C. Tan, and P. Wu, Phys. Rev. B 76, 155435 (2007).

${ }^{18}$ M. Bruno, M. Palummo, S. Osscini, and R. D. Sole, Surf. Sci. 601, 2707 (2007).

${ }^{19}$ P. B. Allen and M. Cardona, Phys. Rev. B 27, 4760 (1983).

${ }^{20}$ J. Li and A. J. Freeman, Phys. Rev. B 74, 075333 (2006).

${ }^{21}$ A. R. Guichard, D. N. Barsic, S. Sharma, T. I. Kamins, and M. L. Brongersma, Nano Lett. 6, 2140 (2006).

${ }^{22}$ U. Landman, R. N. Barnett, A. G. Scherbakov, and P. Avouris, Phys. Rev Lett. 85, 1958 (2000).

${ }^{23}$ T. Markussen, R. Rurali, M. Brandbyge, and A. P. Jauho, Phys. Rev. B 74, 245313 (2006).

${ }^{24}$ T. Markussen, R. Rurali, A. P. Jauho, and M. Brandbyge, J. Comput. Electron. 7, 324 (2008).

${ }^{25}$ R. Rurali, T. Markussen, J. Suñé, and M. Brandbyge, Nano Lett. 8, 2825 (2008).

${ }^{26}$ T. Markussen, A. P. Jauho, and M. Brandbyge, Phys. Rev. B 79, 035415 (2009).

${ }^{27}$ M. Amato, S. Ossicini, and R. Rurali, Nano Lett. 11, 594 (2011).

${ }^{28}$ K. Kobayashi, Phys. Rev. B 69, 115338 (2004).

${ }^{29}$ Y. Cui, X. Duan, J. Hu, and C. M. Lieber, J. Phys. Chem. B 104, 5213 (2000).
${ }^{30}$ D. Sánchez-Portal, P. Ordejón, E. Artacho, and J. M. Soler, Int. J. Quantum Chem. 65, 453 (1997).

${ }^{31}$ J. M. Soler, E. Artacho, J. D. Gale, A. García, J. Junquera, P. Ordejón, and D. Sánchez-Portal, J. Phys.: Condens. Matter 14, 2745 (2002).

${ }^{32}$ C. D. Pemmaraju, I. Rungger, and S. Sanvito, Phys. Rev. B 80, 104422 (2009)

${ }^{33}$ A. Odell, A. Delin, B. Johansson, I. Rungger, and S. Sanvito, ACS Nano 4, 2635 (2010).

${ }^{34}$ N. Troullier and J. L. Martins, Phys. Rev. B 43, 1993 (1991).

${ }^{35}$ A. R. Rocha, V. M. García-Suarez, S. W. Báiley, C. J. Lambert, J. Ferrer, and S. Sanvito, Nat. Mater. 4, 335 (2005).

${ }^{36}$ A. R. Rocha, V. M. García-Suarez, S. Báiley, C. Lambert, J. Ferrer, and S. Sanvito, Phys. Rev. B 73, 085414 (2006).

${ }^{37}$ I. Rungger and S. Sanvito, Phys. Rev. B 78, 035407 (2008).

${ }^{38}$ F. Xu and A. Sadrzadeh, Z. Xu and B. I. Yakobson. J. Appl. Phys. 114, 063714 (2013).

${ }^{39}$ I. K. Robinson, P. A. Bennett, and F. J. Himpsel, Phys. Rev. Lett. 88, 096104 (2002).

${ }^{40}$ J. J. Yeh, J. Hwang, K. Bertness, D. J. Friedman, R. Cao, and I. Lindau, Phys. Rev. Lett. 70, 3768 (1993).

${ }^{41}$ J. K. Tomfohr and O. F. Sankey, Phys. Rev. B 65, 245105 (2002).

${ }^{42}$ C. Cucinotta, I. Rungger, and S. Sanvito, J. Phys. Chem. C 116, 22129 (2012).

${ }^{43}$ W. H. Butler, X.-G. Zhang, T. C. Schulthess, and J. M. MacLaren, Phys. Rev. B 63, 054416 (2001).

${ }^{44}$ M. Paulsson and M. Brandbyge, Phys. Rev. B 76, 115117 (2007). 